\title{
Usefulness of Acceleration Time for Internal Carotid Artery Origin Stenosis
}

\author{
Hirokazu Tamura, RT, ${ }^{1}$ Yasuhisa Akaiwa, $\mathrm{MD}, \mathrm{PhD},{ }^{2}$ and Kiyoshi Onda, $\mathrm{MD}, \mathrm{PhD}^{3}$
}

Calcification of the internal carotid artery (ICA) hinders accurate evaluation of the stenosis by conventional ultrasonography due to acoustic shadow. We examined the relationship between acceleration time (AcT) and ICA origin stenosis. One hundred thrity seven samples (266 vessels) that enforced duplex ultrasonography in our hospital were targeted. The results have shown that there is a significant relationship between AcT and stenosis. AcT of more than 110 msec suggests that the stenosis is more than $60 \%$ by the North American Symptomatic Carotid Endarterectomy Trial (NASCET) method. AcT is thought to be useful for the diagnosis of ICA stenosis with calcification. (*English Translation of J Jpn Coll Angiol 2011; 51: 365-371)

Keywords: acceleration time, internal carotid artery, ultrasonography

\section{INTRODUCTION}

$\mathrm{T}$ he carotid artery stenosis is a risk factor for cerebral infarction. Recently, procedures such as carotid endarterectomy (CEA) and carotid artery stenting (CAS) have been recommended depending on the severity of stenosis. ${ }^{1)}$ Ultrasonography is a simple and noninvasive examination widely used for the diagnosis of carotid artery stenosis. However, apparatus-specific artifacts are occasionally observed, and the evaluation of stenosis with calcification may be hampered by acoustic shadows.

The presence of stenosis is known to make the upstroke of the blood flow velocity waveform gentler on its distal

${ }^{1}$ Department of Medical Radiology, Niigata Neurosurgical Hospital, Niigata, Niigata, Japan

${ }^{2}$ Department of Neurology, Niigata University Medical and Dental Hospital, Niigata, Niigata, Japan

${ }^{3}$ Department of Neurosurgery, Niigata Neurosurgical Hospital, Niigata, Niigata, Japan

Received: June 5, 2013; Accepted: June 5, 2013

Corresponding author: Hirokazu Tamura. Department of Medical Radiology, Niigata Neurosurgical Hospital, 3057 Yamada, Nishi-ku,

Niigata, Niigata 950-1101, Japan

Tel: +81-25-231-5120, Fax: +81-25-231-5130

E-mail: hrkztmr@yahoo.co.jp

*This article is English Translation of J Jpn Coll Angiol 2011; 51:

365-371. side and to prolong the systolic acceleration time (AcT). ${ }^{2)}$ These changes in the flow velocity waveform have been reported to be useful for the diagnosis of stenosis in peripheral arteries and the renal artery. ${ }^{3,4)}$ On ultrasonography of the cervical blood vessels, the diagnosis of stenosis often depends on changes in the flow velocity waveform pattern, and prolonged AcT. In this study, we examined the relationship between $\mathrm{AcT}$ and the severity of stenosis at the origin of the internal carotid artery (ICA).

\section{Materials And Methods}

The materials were 274 vessels of 137 patients (99 males and 38 females aged 35-90 years, with a mean of 66.4 years) who underwent ultrasonography of the cervical blood vessels between June 2007 and April 2010. Patients with a history of aortic stenosis (AS) were excluded from the subjects. Excluding 8 vessels with obstruction of the internal carotid artery, stenosis of the common carotid artery, or stenosis of the brachiocephalic artery, 266 vessels were eventually evaluated.

Ultrasonography was carried out using Vivid7 Dimension (GE Healthcare, Tokyo, Japan) and a linear probe with a central frequency of $9 \mathrm{MHz}$ in the supine position. The percent stenosis was evaluated by ultrasonography using the North American Symptomatic Carotid Endarterectomy 


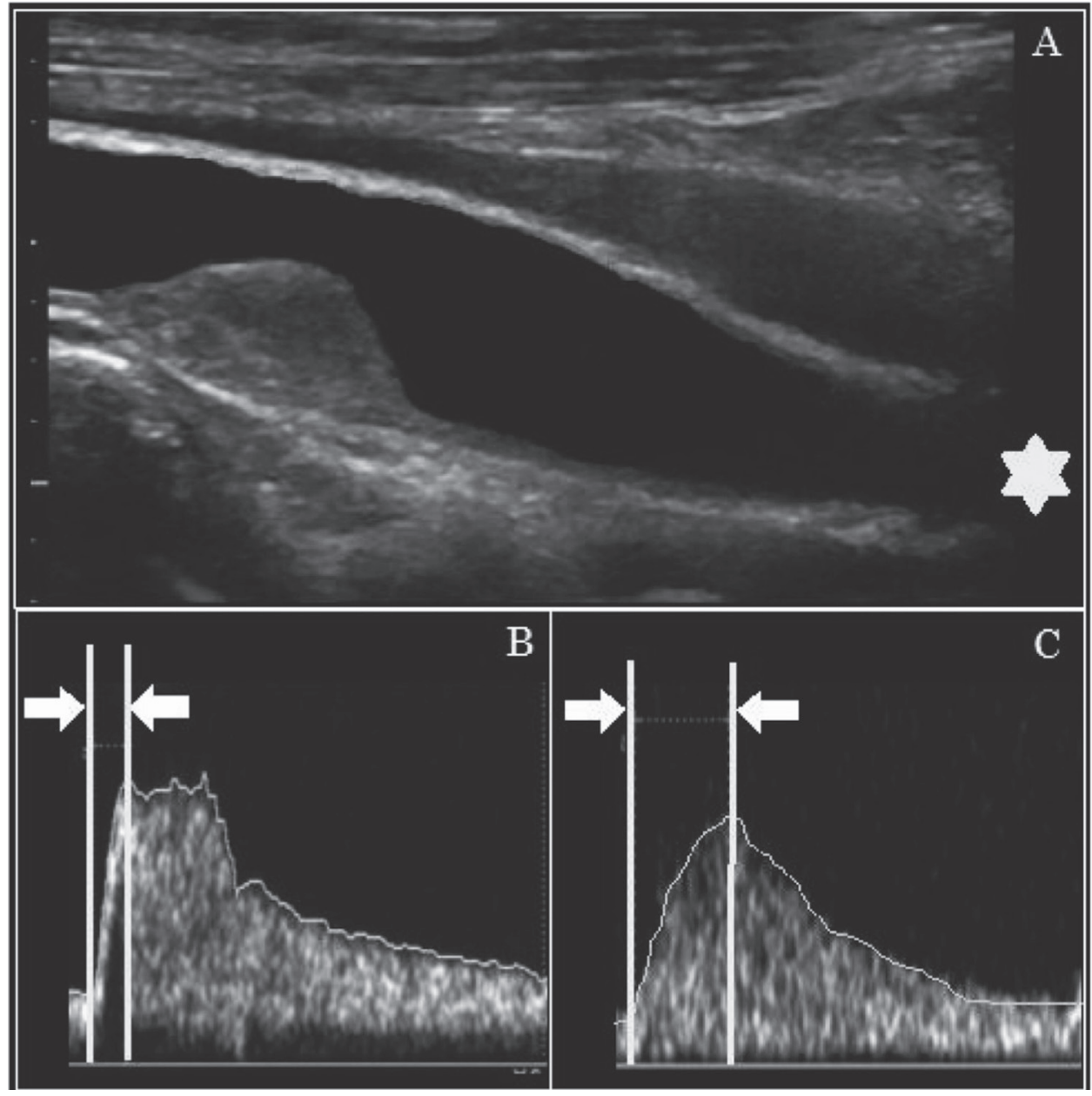

Fig. 1 Measurement methods of acceleration time (AcT). (A) The linear array probe was set in the internal carotid artery (ICA) at $2-3 \mathrm{~cm}$ above the ICA origin. The AcT was measured using the average of 3 heartbeats. (B) The normal Doppler wave-form pattern. (C) Duplex sonogram of a patient with ICA stenosis shows that upstroke is delayed and peak is rounded.

\section{Trial (NASCET) method. ${ }^{5)}$}

The AcT and the flow velocity were measured using the duplex Doppler technique by avoiding curved parts of the vessel. The measurements were performed $2-3 \mathrm{~cm}$ above the carotid bifurcation in patients with no stenosis and $2-3 \mathrm{~cm}$ above the stenosed lesion in patients with stenosis (Fig. 1). The AcT was measured with the ultrasonographic apparatus, and the mean of 3 heartbeats was calculated. The sample volume was $2 / 3$ of the vascular diameter, and the angle of incidence of ultrasound relative to the direction of the blood flow was set at $60^{\circ}$ or less. If the flow velocity waveform showed 2 peaks, the time from the upstroke to the first peak was regarded as the AcT.

Of the 266 vessels, 172 that showed no stenosis were used as a control group, and the mean $+2 \mathrm{SD}$ was calcu- lated as a normal cutoff value. Ninety-four vessels that showed $10 \%$ or more stenosis by the NASCET method were regarded as a stenosis group, and the relationship between the percent stenosis and AcT was evaluated (Fig. 2).

The percent stenosis was also evaluated by angiography (angio-NASCET) in patients who were judged by the attending physician to require the examination and consented to it. Angiography was performed using Advantx UNV (GE Healthcare, Japan) and Allura Xper FD 20/10 (Royal Philips Electronics, Amsterdam, Netherlands). Correlations between 2 variables were examined using Pearson's correlation coefficient. Comparisons between the 2 groups were made with Mann-Whitney's U-test at the $\mathrm{P}<0.05$ level of significance. Concerning the percent stenosis and AcT, the sensitivity and specificity 


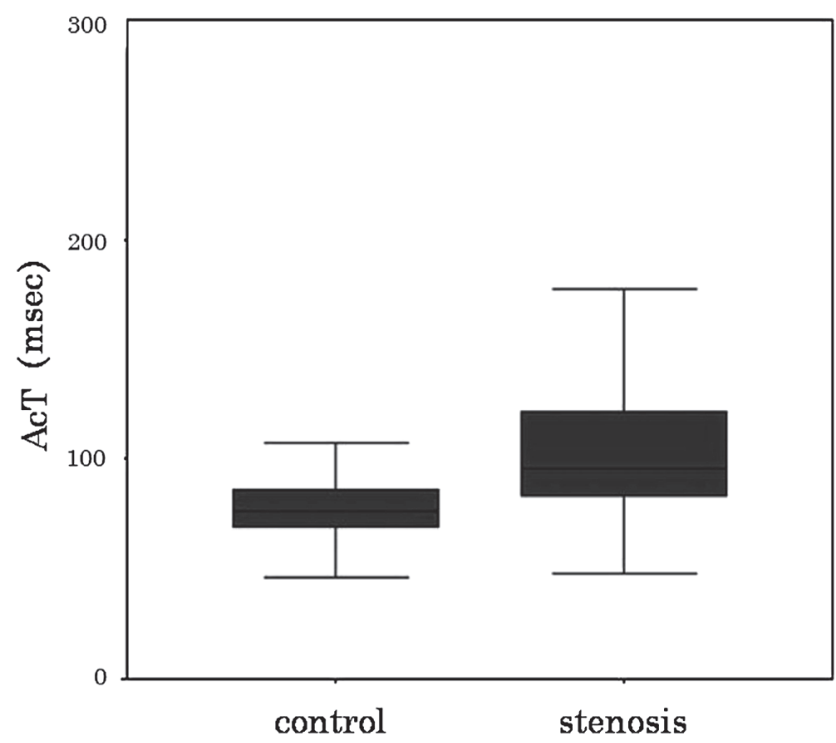

Fig. 2 Comparison of acceleration time in control group $(<$ NASCET $10 \%)$ and the stenosis group ( $\geq$ NASCET 10\%). NASCET: North American Symptomatic Carotid Endarterectomy Trial

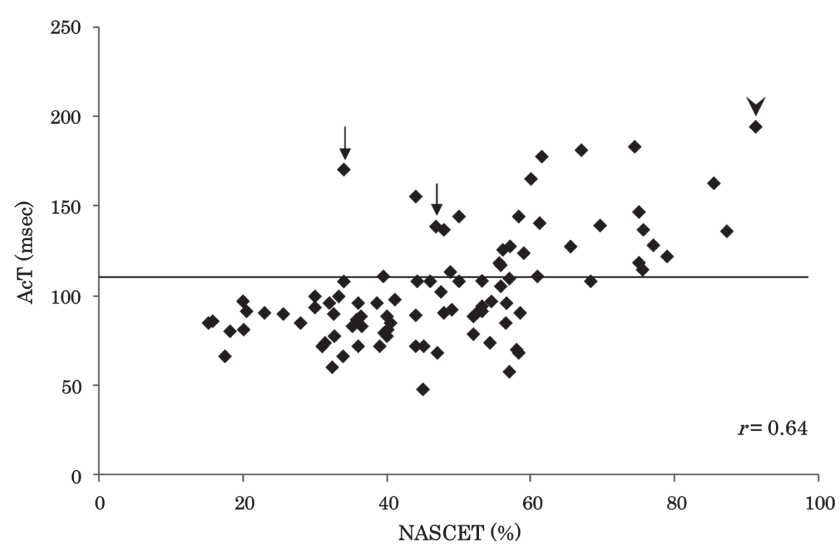

Fig. 3 Relationship between degree of stenosis by the duplex NASCET method and AcT $(r=0.64, \mathrm{P}<0.01)$. AcT has extended by the near occlusion of ICA (arrow head) and the severe kink of ICA (arrow). NASCET: North American Symptomatic Carotid Endarterectomy Trial; AcT: acceleration time; ICA: internal carotid artery

were calculated using the receiver operating characteristic (ROC) curve.

\section{Results}

\section{Relationship between the AcT and the degree of ste- nosis by ultrasonography}

The mean AcT was $79.0 \pm 15.5 \mathrm{msec}$ in the control group, and the mean $+2 \mathrm{SD}$, which was $110 \mathrm{msec}$, was set as the cutoff value.

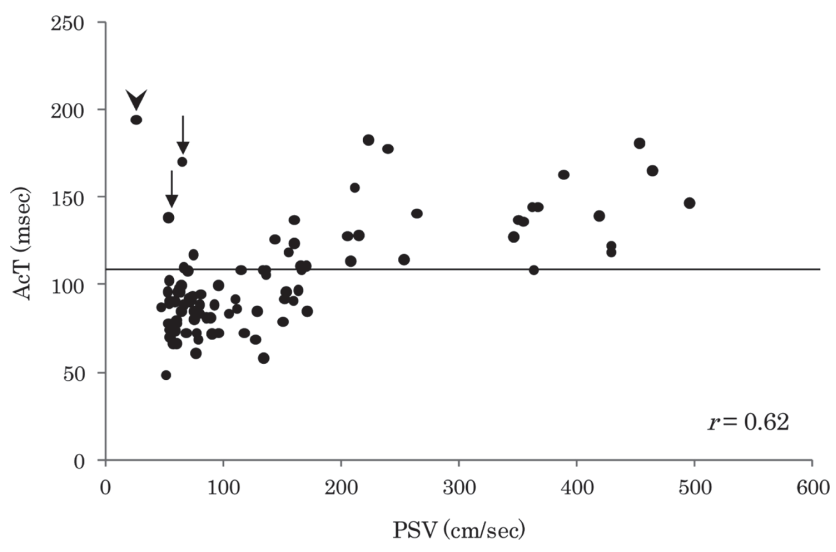

Fig. 4 Relationship between PSV of ICA origin and AcT $(\mathrm{r}=0.62, \mathrm{P}<0.01)$. AcT has extended by the near occlusion of ICA (arrow head) and the severe kink of ICA (arrow). PSV: peak systolic velocity; ICA: internal carotid artery, AcT: acceleration time

In the stenosis group (94 vessels), the AcT and the percent stenosis by ultrasonography showed a significant correlation $(\mathrm{r}=0.64, \mathrm{P}<0.01)$. Also, with the above cutoff value, $60 \%$ stenosis by the NASCET method could be predicted (Fig. 3). A significant correlation was also noted between the peak systolic velocity (PSV) and AcT $(\mathrm{r}=0.62, \mathrm{P}<0.01)$. With the above cutoff value, stenosis with a PSV of $170 \mathrm{~cm} / \mathrm{sec}$ could be predicted (Fig. 4).

The AcT was prolonged in some vessels showing no increase in the PSV, but this was due to meandering observed between the carotid bifurcation and measurement site (Figs. 3 and 4; arrows) and near-occlusion of the ICA (Figs. 3 and 4; arrowheads).

When an AcT of $110 \mathrm{msec}$ was set as the cutoff value, the sensitivity and specificity for NASCET $\geq 50 \%$ were $58.1 \%$ and $88.2 \%$, those for NASCET $\geq 60 \%$ were $94.7 \%$ and $82.7 \%$, and those for NASCET $\geq 70 \%$ were $100 \%$ and $75.9 \%$, respectively (Fig. 5).

\section{Comparison with the results of angiography}

Angiography was performed in 11 patients (11 vessels). The mean AcT was $138.5 \pm 26.3 \mathrm{msec}$ in the vessel on the affected side and significantly prolonged compared with $91.0 \pm 24.7 \mathrm{msec}$ on the opposite side $(\mathrm{P}<0.01)$. The percent stenosis determined by angiography was also correlated with the AcT $(r=0.70, \mathrm{P}<0.01)$ (Fig. 6).

A case in which the degree of stenosis could not be evaluated by ultrasonography due to calcification of the vascular wall is presented (Fig. 7). The AcT of the ICA was prolonged to $122 \mathrm{msec}$, and $60 \%$ or severer stenosis 

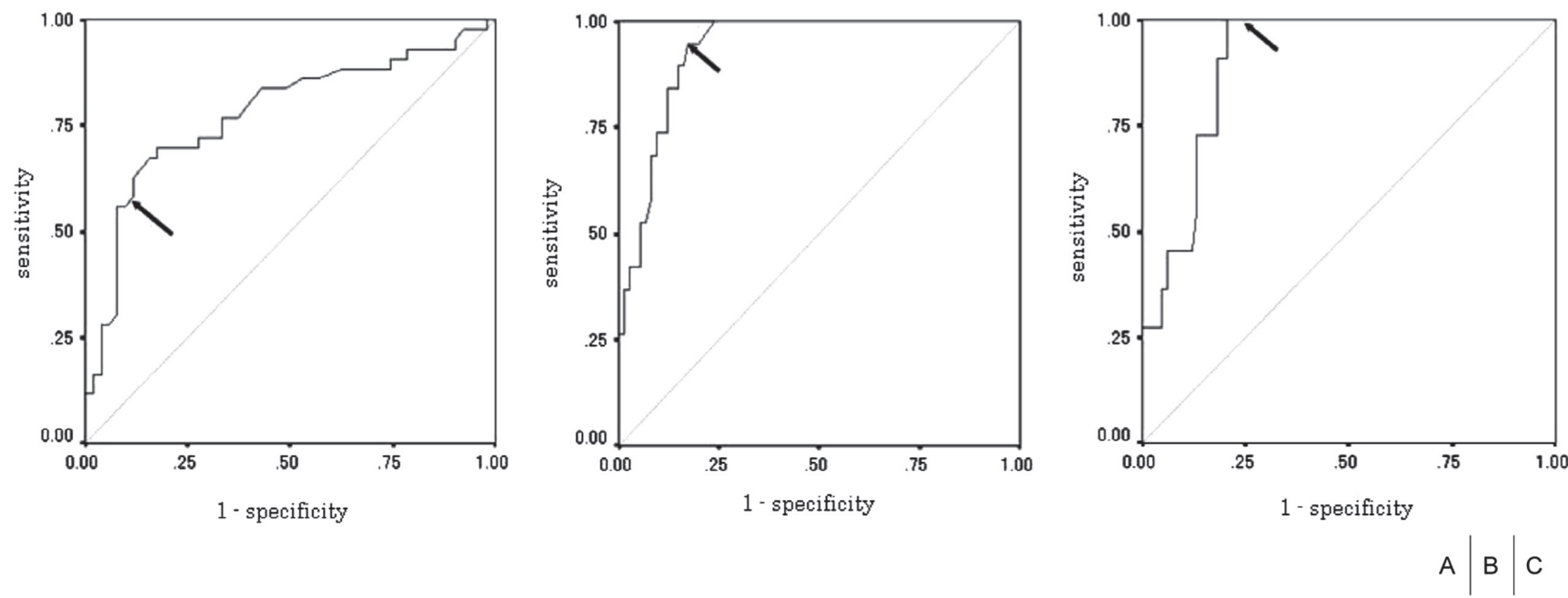

Fig. 5 Sensitivity and Specificity of AcT. (A) In the receiver operating characteristic (ROC) curve, when the cutoff value of the AcT was set at $110 \mathrm{msec}$, the sensitivity was $58.1 \%$ and the specificity was $88.2 \%$ for an ICA stenosis of more than $50 \%$ by the duplex NASCET method. (B) The sensitivity was $94.7 \%$ and the specificity was $82.7 \%$ for an ICA stenosis of more than $60 \%$. (C) The sensitivity was $100 \%$ and the specificity was $75.9 \%$ for an ICA stenosis of more than $70 \%$. AcT: acceleration time; ICA: internal carotid artery; NASCET: North American Symptomatic Carotid Endarterectomy Trial

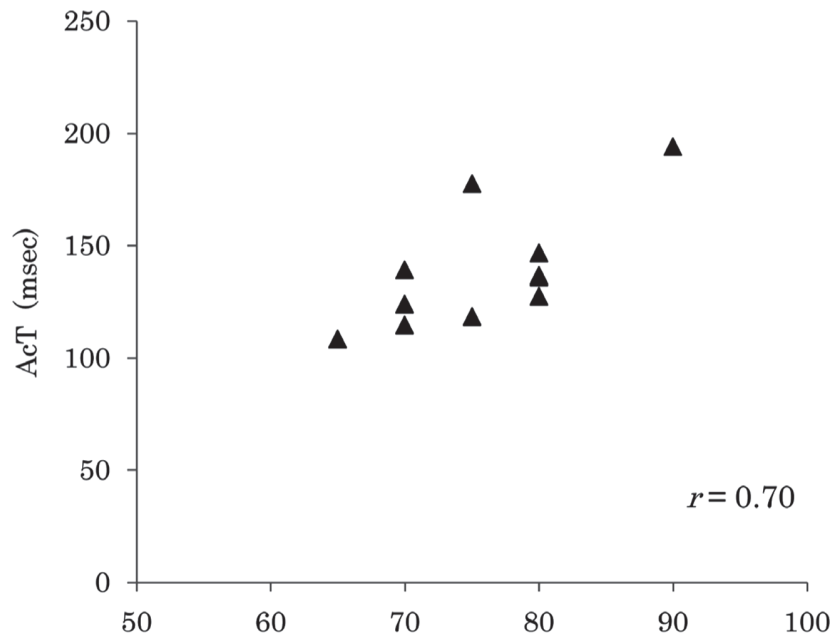

Fig. 6 Relationship between degree of stenosis by the angiographic NASCET method and AcT $(r=0.70, \mathrm{P}<0.01)$. NASCET: North American Symptomatic Carotid Endarterectomy Trial; AcT: acceleration time

was suspected. Angiography of the same site showed $80 \%$ stenosis at the origin of the ICA, as expected from the AcT.

\section{Discussion}

CEA and CAS are considered as treatments for carotid artery stenosis depending on its severity. The NASCET method based on angiography is widely used for evaluation of the percent stenosis, ${ }^{6}$ but complications accompanied by neurological symptoms occur in about $4 \%$ of patients undergoing angiography, ${ }^{7}$ and there is also concern over adverse effects of the contrast agent.

On the other hand, as ultrasonography is a simple and noninvasive examination, it is routinely used in clinical practice. The NASCET method, European Carotid Surgery Trial (ECST) method, area stenosis rate, and PSV are used to evaluate the degree of stenosis by ultrasonography. Evaluation using the PSV, in particular, is known to be highly reproducible ${ }^{8)}$ and correlated with the angiographic NASCET percent stenosis, and PSVs of $150 \mathrm{~cm} / \mathrm{sec}$ or higher and $200 \mathrm{~cm} / \mathrm{sec}$ or higher suggest NASCET $50 \%$ or more and $70 \%$ or more stenosis, respectively. ${ }^{3,9)}$ However, in calcified stenotic lesions, measurement of the PSV is occasionally difficult by ultrasonography due to acoustic shadows.

We evaluated the relationship between the AcT and stenosis at the origin of the ICA. On vascular ultrasonography, if there is a stenotic lesion on the proximal side, the upstroke of the blood flow velocity waveform is known to become gentler, and the AcT to be prolonged, on the distal side of the lesion. ${ }^{2)}$ In this study, the AcT was significantly correlated with both ultrasonographic NASCET percent stenosis and PSV, and an AcT of $110 \mathrm{msec}$ or higher suggested stenosis with a NASCET 


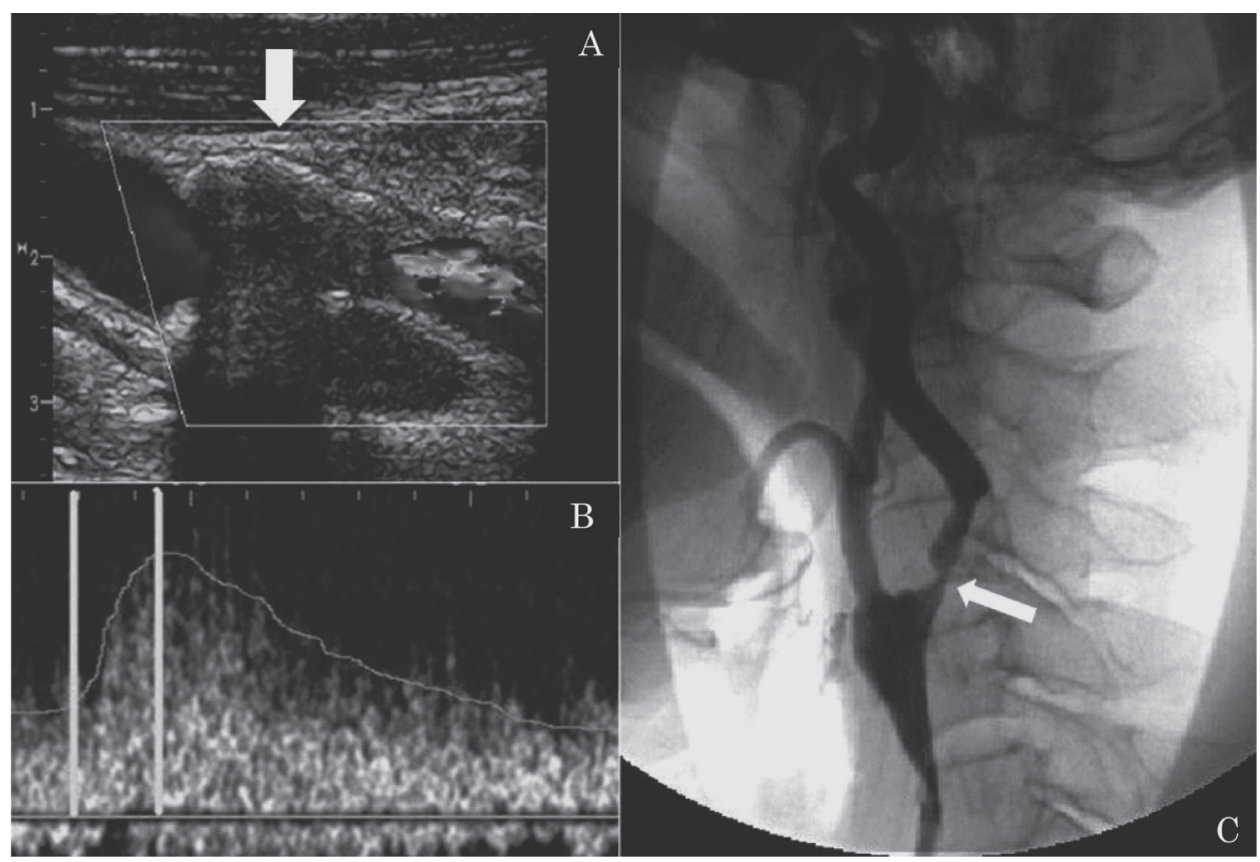

Fig. 7 (A) Ultrasonography shows ICA origin. The evaluation of ICA origin is difficult due to the calcification of the vessel wall (arrow). (B) Doppler waveform shows post-stenotic pattern. AcT is $122 \mathrm{msec}$. (C) Lateral view of a left common carotid angiogram shows $80 \%$ ICA stenosis by the NASCET method (arrow). ICA: internal carotid artery; AcT: acceleration time; NASCET: North American Symptomatic Carotid Endarterectomy Trial

$60 \%$ or above and a PSV of $170 \mathrm{~cm} / \mathrm{sec}$ or above. In the ROC curve, when an AcT of $110 \mathrm{msec}$ was set as the cutoff value, both the sensitivity and specificity for the detection of NASCET $60 \%$ or severer stenosis were high. NASCET $60 \%$ is a criterion for recommending CEA for asymptomatic carotid artery stenosis. ${ }^{1)}$ Therefore, the AcT is considered to serve as a reference for the selection of surgical treatment.

$\mathrm{AS}$ is known to be a factor prolonging the AcT in the ICA. In severe AS, the AcT has been reported to be prolonged in the common carotid artery (CCA), ICA, and vertebral artery (VA). ${ }^{10)}$ Takekawa, et al. reported that these conditions can be excluded using the AcT ratio, calculated by dividing the AcT of the ICA by the AcT of the ipsilateral CCA. They also compared the AcT ratio and degree of stenosis, and reported that an AcT ratio of 1.5 or higher suggests NASCET $50 \%$ or severer stenosis and that an AcT of 2.0 or higher suggests NASCET $70 \%$ or severer stenosis. ${ }^{11)}$ In our study, we did not perform transthoracic echocardiography in all patients and did not precisely evaluate AS, but patients with severe AS, confirmed by checking with the chart or attending physician, were excluded. With a check of the history for AS, evaluation of stenosis at the origin of the ICA is consid- ered to be possible even by the AcT alone.

The presence of stenosis on the proximal side makes the upstroke of the blood flow velocity waveform gentler. Therefore, evaluation of the acceleration, not the acceleration time, of the flow velocity during systole is also considered, but this problem will be tackled in the future. Also, it is necessary to pay attention that the AcT is considered to be prolonged in patients with a bend before the measurement site.

The value used as the cutoff was a reference value at our hospital, and so a cutoff value for general use should be determined by evaluating various factors involved in prolongation of the AcT. As this study aimed to compare the AcT with percent stenosis based on ultrasonography, it was also necessary for a definitive diagnosis by angiography. However, as the validity of the AcT could be confirmed by comparison with the results of angiography, the AcT may contribute to the diagnosis of significant stenosis on ultrasonography.

\section{Conclusion}

The AcT is one of the parameters that can be easily determined by ultrasonography. Measurement of the AcT 
in the ICA, with even calcified plaque, was suggested to be useful for evaluation of the presence and severity of stenosis at the origin of the ICA.

\section{ACKnowledgments}

The authors express their sincere gratitude to Drs. Hiroyuki Arai, Kazunori Yamazaki, and Teruo Miyakawa of Niigata Neurosurgical Hospital and Brain Research Center and Dr. Hidehiro Takekawa of the Stroke Section, Department of Neurology, Dokkyo Medical University for their generous cooperation.

\section{Disclosure Statement}

The authors have no conflict of interest to declare.

\section{REFERENCES}

1) Japanese Guideline for the Management of Stroke. The Joint Committee on Guideline for the Management of Stroke. Kyowa Kikaku, Tokyo, 2009, 227-229. (in Japanese)

2) The Japan Academy of Neurosonology. Carotid Ultrasound Examination. 2006; 19: 40-67. (in Japanese)

3) Hirai T, Higashiura W, Sakaguchi S, et al. Ultrasonography of the Peripheral Arterial Disease-Recent Advances and Clinical Application-. J Jpn Coll Angiol 2004; 44: 727-34. (in Japanese)
4) Burdick L, Airoldi F, Marana I, et al. Superiority of acceleration and acceleration time over pulsatility and resistance indices as screening tests for renal artery stenosis. J Hypertens 1996; 14: 1229-35.

5) Beneficial effect of carotid endarterectomy in symptomatic patients with high-grade carotid stenosis. North American Symptomatic Carotid Endarterectomy Trial Collaborators. N Engl J Med 1991; 325: 445-53.

6) Cremonesi A, Setacci C, Bignamini A, et al. Carotid artery stenting: first consensus document of the ICCS-SPREAD Joint Committee. Stroke 2006; 37: 2400-9.

7) Hankey GJ, Warlow CP, Sellar RJ. Cerebral angiographic risk in mild cerebrovascular disease. Stroke 1990; 21: 209-22.

8) Sabeti S, Schillinger M, Mlekusch W, et al. Quantification of internal carotid artery stenosis with duplex US: comparative analysis of different flow velocity criteria. Radiology 2004; 232: 431-9.

9) Koga M, Kimura K, Minematsu K, et al. Diagnosis of internal carotid artery stenosis greater than $70 \%$ with power Doppler duplex sonography. AJNR Am J Neuroradiol 2001; 22: 413-7.

10) O'Boyle MK, Vibhakar NI, Chung J, et al. Duplex sonography of the carotid arteries in patients with isolated aortic stenosis: imaging findings and relation to severity of stenosis. AJR Am J Roentgenol 1996; 166: 197-202.

11) Takekawa $H$, Asakawa $Y$, Lee $T$, et al. Usefulness of acceleration time for assessment of stenosis in the extracranial internal carotid artery. Neurosonology 2009; 22: 79-82. (in Japanese) 